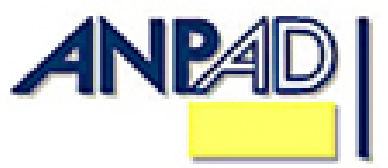

Disponível em

http://www.anpad.org.br/rac

RAC, Curitiba, v. 15, n. 1, art. 7, pp. 126-137, Jan./Fev. 2011

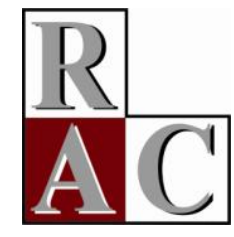

\title{
CEOs e Composição do Conselho de Administração: a Falta de Identificação Pode Ser Motivo para Existência de Teto de Vidro para Mulheres no Brasil?
}

\author{
CEOs and Board Composition: Can the Lack of Identification Be a Reason for Glass \\ Ceilings in Brazil?
}

Regina Madalozzo *

E-mail: reginam@insper.edu.br Insper Instituto de Ensino e Pesquisa

São Paulo, SP, Brasil.

\footnotetext{
* Endereço: Regina Madalozzo

Rua Quatá, 300, 4º andar, São Paulo/SP, 04546-042.
}

Copyright (C) 2011 RAC. Todos os direitos, até mesmo de tradução, são reservados. É permitido citar parte de artigos sem autorização prévia, desde que seja identificada a fonte. 


\title{
Resumo
}

O aumento significante da participação feminina na força de trabalho não impediu que permanecessem as diferenças com relação aos salários e à igualdade de tratamento nas promoções com relação ao gênero. Segundo a teoria da divisão do trabalho dentro da família, de acordo com as vantagens relativas de cada membro do casal (Becker, 1965), a diferença salarial e de promoção pode implicar menor investimento por parte das mulheres em seu aperfeiçoamento profissional. Neste trabalho, utilizamos uma base de dados inédita, referente a 370 empresas, que possibilita a investigação sobre a existência de teto de vidro para as mulheres no Brasil. Utilizando a metodologia empírica de probit, que permite a análise de variáveis binárias qualitativas, como a presença de uma mulher ou um homem no cargo mais elevado de uma empresa, concluiu-se neste estudo que existe uma relação entre a dificuldade da promoção de mulheres e a existência de um Conselho de Administração para ela. Com base na análise de Westphal e Fredrickson (2001), pode-se dizer que os resultados encontrados mostram que o Conselho de Administração busca escolher um CEO (do inglês Chief Executive Officer) que represente ao máximo seu próprio perfil de atuação e que, sendo o Conselho majoritariamente masculino, indivíduos do sexo feminino sofrem maior resistência à promoção.

Palavras-chave: teto de vidro; probit; promoção.

\begin{abstract}
The significant growth of female labor market participation did not resolve the gender-related differences concerning wages and promotion. According to the family division of work theory, a comparative advantage between the couple would determine the home and market division of work (Becker, 1965). The wage gap and promotion rates differential could imply less investment by women in their professional background. In the present work, we use a unique dataset of 370 companies that enable the testing of the glass ceiling phenomenon for women in Brazil. Using the empirical probit methodology that enables a qualitative binary analysis of the major occupation of men or women in the companies, the main conclusion of this study is related to the complexity of female promotion if there is a Board of Administration at the company level. Following the analysis of Westphal and Fredrickson (2001), our results show that the Board usually chooses a CEO that better represents its own profile and, being mostly composed of males, there is greater resistance to female promotion.
\end{abstract}

Key words: glass ceiling; probit; promotion. 


\section{Introdução}

O Brasil, seguindo a tendência mundial, apresentou significativo aumento da participação feminina no mercado de trabalho nas últimas décadas. Segundo dados do IBGE (Instituto Brasileiro de Economia e Estatística), menos de 30\% das mulheres participavam do mercado de trabalho no final da década de 1970 e, em 2007, esse índice já ultrapassava 50\% ${ }^{(1)}$. Ao mesmo tempo, verifica-se a diminuição da diferença salarial entre os gêneros, passando de 33\% em 1978 para 15,40\% em 2007 (Madalozzo, 2009). Entretanto, ainda persiste a discussão a respeito da baixa entrada das mulheres em cargos elevados nas empresas em que trabalham. Segundo pesquisa realizada pelo Grupo Catho, empresa especializada em Recursos Humanos, em 2007, somente 8\% dos presidentes de empresas eram do sexo feminino. Seria essa uma evidência de teto de vidro nas empresas que atuam no Brasil?

A discussão sobre o efeito teto de vidro (glass ceiling) iniciou-se com Morrison, White, Velsor e The Center for Creative Leadership (1987) e incentivou a criação de uma comissão, criada em 1991, pela Secretaria do Trabalho nos Estados Unidos. Naquela ocasião, o governo americano buscava uma forma de regulamentar o mercado com o fim de reduzir barreiras que artificialmente impedissem as mulheres de aproveitarem oportunidades de carreira e de promoção da mesma forma que os homens. A denominação de teto de vidro se deve ao fato de que a promoção interna é responsabilidade dos gestores da empresa, e os critérios para tal não são necessariamente públicos, nem mesmo para os membros internos da empresa, representando uma barreira intransponível e invisível, mas perceptível na análise de progressão na carreira. Nas eleições americanas de 2008, a discussão sobre a diferença de tratamento dos meios de comunicação e dos eleitores com relação a então Senadora Hillary Clinton e seu adversário nas primárias, Barack Obama, trouxe novamente a questão do teto de vidro à tona (Guimarães, 2008).

Pesquisas internacionais não chegaram a um consenso sobre a existência de uma barreira invisível para a promoção das mulheres. Jones e Makepeace (1996) utilizam dados de uma instituição financeira, entre os quais encontram indícios de que, para a promoção de mulheres, os critérios são mais rígidos e elevados do que para a promoção de homens. Blau e Devaro (2007) têm conclusão semelhante, sugerindo que a promoção é menos provável para as mulheres do que para os homens, mas apresentam resultados mostrando que a equiparação salarial em determinadas carreiras já ocorre. Já Powell e Butterfield (1994), utilizando dados de uma organização federal americana, mostram que não existe diferencial de promoção entre homens e mulheres para o nível hierárquico mais elevado dessa instituição.

Neste trabalho, no lugar de utilizarmos dados de uma só empresa, como é mais comum na literatura, usaremos dados de 370 empresas brasileiras, pesquisadas pelo instituto de pesquisa Sensus em 2007. A partir das respostas colhidas, analisaremos quais fatores explicam probabilidades diferentes entre os gêneros para que ocupem o posto mais alto da empresa, denominado CEO (Chief Executive Officer ou, traduzindo, Diretor Executivo) ${ }^{(2)}$. Nossos resultados apontam que a existência de um Conselho de Administração diminui significativamente as chances das mulheres de serem escolhidas para o cargo de CEO. Tal conclusão está em concordância com pesquisas anteriores, como Westphal e Fredrickson (2001). Segundo os autores, pode-se afirmar que a escolha do CEO por parte do Conselho de Administração reflete não somente a experiência e a capacidade do indivíduo, mas também sua similaridade com o perfil do Conselho que o escolheu. Assim, a existência de conselhos majoritariamente masculinos diminui a chance de escolha de uma CEO do sexo feminino.

O artigo está organizado da seguinte forma: na seção seguinte, apresentamos alguns estudos relacionados ao tema. A seção 3 descreve os dados e apresenta as análises descritivas, e é seguida por uma seção dedicada aos resultados empíricos da análise. Por fim, a seção 5 apresenta as conclusões deste trabalho e as direções para pesquisas futuras sobre o mesmo tema. 


\section{Revisão Bibliográfica}

Desde o final da década de 1980, a possibilidade de existência de teto de vidro para as mulheres no mercado de trabalho é discutida. Kaufman e Hotchkiss (2003) apresentam dados americanos que mostram a baixa representatividade das mulheres em cargos de alto escalão. Uma das razões alegadas no passado para esse quadro era a pouca experiência das mulheres no mercado de trabalho. Entretanto, a inserção feminina no mercado de trabalho americano teve alto crescimento na década de 1950. Seria esperado que meio século como mão de obra fosse suficiente para eliminar essa possibilidade, mas o que se verifica é a persistência do efeito. Dessa forma, muitos estudos se concentram em explicar as motivações para uma barreira invisível e dificilmente transponível para a ascensão profissional feminina.

Jones e Makepeace (1996) tiveram acesso aos dados de uma grande instituição financeira da Grã-Bretanha. Com base em dados de mais de 4.000 funcionários em tempo integral desta instituição, que utilizam o mercado de trabalho interno como potencial de promoção, os autores constataram que as mulheres não recebiam tratamento igualitário com relação aos homens. Como a maternidade, por motivos físicos, representa um fardo mais pesado para as mulheres, é natural que tenha também uma influência importante no mercado de trabalho para as trabalhadoras em todos os níveis, pois impede a mulher de ir trabalhar por determinado período de tempo e uma consequente perda de experiência no mercado, que poderia conduzir à promoção. Um dos grandes motivos para o diferente tratamento entre homens e mulheres, nesse caso, era a falta de uma licença maternidade que permitisse o afastamento por tempo determinado e o retorno da mulher após o parto e a amamentação para a mesma função que exercia anteriormente. A impossibilidade de ter uma garantia de retorno à empresa após ter filhos implicaria um investimento menor das mulheres das empresas em sua qualificação e, consequentemente, um índice menor de promoção para as profissionais do sexo feminino.

O impacto positivo na participação das mães no mercado de trabalho devido à licença maternidade também foi constatado por Berger e Waldfogel (2004). Utilizando dados da National Longitudinal Survey of Youth, um painel com dados da população americana para o período de 19881996, os autores mostram que mães que já participavam do mercado de trabalho e tinham empregos nos quais seu retorno ao trabalho era garantido após a licença maternidade retornavam a seus postos de trabalho em menos de 12 meses. Já para aquelas que não trabalhavam antes do parto ou estavam empregadas em empresas que não ofereciam esse benefício, o retorno era mais demorado. A importância de um retorno mais rápido ao mercado de trabalho após ter filhos está relacionada à menor perda de experiência por afastamento de suas atividades no mercado (Gordo, 2009; Napari, 2010).

Em termos de modelagem econômica, Bjerk (2008) mostra que a existência de teto de vidro não está somente relacionada à discriminação contra um gênero ou uma etnia, mas também à dificuldade de avaliação de habilidades. Quanto mais escassas as oportunidades de mostrar características benéficas ao novo cargo e quanto menor a crença com relação aos sinais emitidos pelos indivíduos, maior a chance de existir uma barreira invisível para a promoção de determinados grupos demográficos (mulheres, por exemplo). Essa dificuldade, assim, não está unicamente conectada com um suposto preconceito.

Hultin (2003) exemplifica essa conclusão, ao mostrar que homens que optaram por profissões consideradas femininas, como trabalhar na área de recursos humanos ou como professor de ensino fundamental ou médio, por exemplo, têm maior probabilidade de promoção do que mulheres na mesma profissão. Pode-se dizer, portanto, que não é o tipo de profissão que as mulheres escolhem que causa uma demora na promoção, mas sim o fato de serem mulheres. Emitir um sinal mais favorável de permanência no emprego e a maior possibilidade de experiência anterior no cargo ou em empregos prévios aumenta a chance de promoção para indivíduos do sexo masculino, mesmo quando alocados em empregos pouco aprazíveis para seu gênero. 
Wolfers (2006) relaciona os retornos das ações ao gênero do CEO. Sua abordagem busca verificar se existe diferença do valor estimado das empresas e o valor de venda efetivo a partir do gênero do CEO. Com dados da S\&P (Standard \& Poors, agência de ratings, divulgação de índices e informações a respeito de empresas) no período de 1992-2004, o autor não encontra diferenças sistemáticas que sejam fundamentadas no sexo do CEO, mas levanta a possibilidade de estar usando testes estatísticos de baixo poder, que tornariam suas conclusões tendenciosas nesse sentido.

Lee e James (2007) analisam anúncios de trocas de CEOs na década de 1990 e mostram que aqueles relacionados à entrada de um CEO do sexo feminino têm impactos significativamente mais negativos do que os relacionados à entrada de um CEO do sexo masculino. Além disso, os anúncios de CEOs do sexo feminino dão ênfase justamente ao gênero do profissional e à necessidade de representatividade do gênero na empresa, não à capacidade ou aos antecedentes profissionais e qualificadores que o levaram a ser eleito CEO na empresa. O mesmo, porém, não é verificado quando o anúncio é da entrada de um CEO do sexo masculino.

Em resumo, a análise feita para países desenvolvidos aponta a tendência de estagnação na recuperação das mulheres em face dos homens no mercado de trabalho. Por ocasião da entrada mais efetiva das mulheres no mercado, que se deu na metade do século passado, coincidindo com o final da $2^{\circ}$. Grande Guerra, verificava-se grande diferença salarial e de condições de trabalho, quando se comparavam os dois gêneros. Ao longo da segunda metade do século XX, estas diferenças foram diminuindo, com mulheres conseguindo promoção para alguns cargos de chefia e liderança. Entretanto, foi a partir da década de 1990 que estudos apontam que, mesmo com formação e experiência compatíveis entre homens e mulheres, permanece uma diferença tanto salarial quanto de promoção dentro das empresas, quando se comparam os gêneros, conforme os estudos anteriormente citados demonstram. Esta diferença de tratamento para homens e mulheres tanto reforça a ideia de dominação de um gênero sobre o outro, como pode conduzir a um retrocesso em termos de conquistas por parte das mulheres por elas verificarem que o investimento que fazem em sua capacitação não tem retorno suficiente no mercado, em face desta desigualdade de tratamento (Bourdieu, 2009).

No Brasil, segundo o presente levantamento de dados, poucos estudos acadêmicos estão preocupados em analisar uma possível dificuldade de promoção para as mulheres. Diversos estudos abordam a diferença de salários de homens e de mulheres (Carvalho, Firpo, \& Gonzaga, 2006; Giuberti \& Menezes, 2005); mas somente Santos e Ribeiro (2006) analisam a questão da promoção. Utilizando a metodologia de regressões quantílicas e dados da Pesquisa Nacional de Amostra por Domicílios (PNAD) de 1999, os autores comparam os rendimentos de diferentes quantis, a partir das características da distribuição de salários de homens e mulheres no Brasil. Os resultados do estudo apontam tanto para uma valorização maior das características femininas na cauda inferior da distribuição - salários menores - como para a existência de preconceito na cauda superior, indicando a existência de teto de vidro para as mulheres no mercado brasileiro.

Uma das explicações para a baixa frequência de estudos relacionados à questão do teto de vidro no Brasil é a dificuldade para encontrar dados que possibilitem tal abordagem. No presente estudo, temos a oportunidade de conhecer algumas características das empresas e dos seus CEOs, permitindo um foco direcionado a essa questão.

\section{Base de Dados e Descrição da Amostra}

Este artigo utiliza como base de dados o resultado da pesquisa Empresas no Brasil, realizada pela empresa Sensus Pesquisa e Consultoria em 2007. O período de campo da pesquisa foi de 10 de setembro a 18 de outubro daquele ano. Os pesquisadores da empresa Sensus conduziram 370 entrevistas por telefone nas cinco regiões geográficas brasileiras, com pessoas jurídicas escolhidas de forma aleatória no Relatório Anual da Gazeta Mercantil. A amostra foi representativa da população de empresas no Relatório da Gazeta Mercantil, de onde foram retirados os nomes, telefones e dados 
contábeis das empresas a serem analisadas. Na pesquisa, foram coletados dados relativos a questões de gestão, precificação de mercadorias e custos empresariais. Após a coleta, os dados financeiros divulgados anualmente no Relatório da Gazeta Mercantil foram devidamente agregados à amostra do campo.

A Tabela 1 apresenta os principais dados descritivos da amostra.

Tabela 1

\section{Descrição das Empresas}

Média

(desvio padrão)

Painel I: Dados relativos à empresa

Receita Líquida (em milhares de reais, ano)

Setor (em \% da amostra):

Agronegócios

11,4

Comércio

17,3

Indústria

21,9

Serviços

49,5

Tempo de existência da empresa (em anos)

$(23,81)$

Painel II: Dados relativos à existência de Conselho de Administração e Controle Acionário

Tem um Conselho Administrativo (\% da amostra)

Percentual do controle acionário da empresa por seus executivos

Empresas com controle acionário $100 \%$ em poder de seus executivos (\% da amostra)

39,46

Número de Observações 370

A Tabela 1 apresenta os dados em duas categorias distintas. No Painel I, dados a respeito da empresa de forma geral são expostos e, no Painel II, questões relativas à gestão da empresa são apresentadas.

No Painel I, verificamos que as empresas investigadas têm um faturamento médio de aproximadamente 190 milhões de reais por ano, e estão atuando no mercado por 32 anos em média ${ }^{(3)}$. Com relação ao setor de atuação, concentram-se no setor de serviços $(49,5 \%)$ seguido pela indústria $(21,9 \%)$, pelo comércio $(17,3 \%)$ e por agronegócios (somente 11,4\%).

No Painel II, observamos que 64\% das empresas têm um Conselho Administrativo e aproximadamente $40 \%$ têm o controle acionário totalmente em poder de seus executivos. Em média, $62 \%$ das ações estão em poder dos executivos da empresa, indicando grau elevado de concentração de poder decisório nas mãos de seus gestores.

Além dos dados das empresas, podemos analisar também o perfil da pessoa que ocupa o cargo mais elevado em sua hierarquia ${ }^{(4)}$. A Tabela 2 apresenta estes números. Somente $8,55 \%$ das empresas têm como seu CEO uma mulher. A idade média dessas mulheres é de 52 anos, e, em média, elas estão na empresa por aproximadamente 16 anos e no cargo há 10 anos.

A maior parte desses indivíduos tem estudo de nível superior completo no mínimo, e somente $10 \%$ deles declaram terem cursado o ensino fundamental ou médio. É interessante ressaltar que $38 \%$ 
dos CEOs completaram alguma pós-graduação (27\%), mestrado (5\%) ou doutorado $(7,4 \%)$. Comparando os resultados por gênero, não verificamos diferenças significativas nas amostras, conforme é possível verificar na última coluna da Tabela 2, na qual apresentamos o resultado do teste de médias entre os dados de homens e mulheres e todos são diferentes estatisticamente no nível de 5\%.

A partir dessas informações, separamos dados relevantes das empresas que poderiam afetar positiva ou negativamente a probabilidade de uma mulher ocupar o cargo de CEO nas mesmas ${ }^{(5)}$. Utilizaremos um modelo Probit para inferir esses efeitos.

Tabela 2

Características dos CEOs

\begin{tabular}{lcccc}
\hline & Total ${ }^{1}$ & Homens & Mulheres & $\begin{array}{c}\text { Diferença entre } \\
\text { Médias }\end{array}$ \\
\hline Idade & 51,57 & 52,22 & 49,69 & 2,056 \\
& $(10,66)$ & $(10,80)$ & $(12,23)$ & $(2,07)$ \\
Educação (em percentual): & & & & \\
Ensino Fundamental ou Médio & 0,104 & 0,105 & 0,103 & 0,001 \\
Superior & 0,499 & 0,497 & 0,518 & $-0,060)$ \\
& & & & $(0,097)$ \\
Pós-Graduação & 0,272 & 0,268 & 0,310 & $-0,042$ \\
& & & & $(0,087)$ \\
Mestrado & 0,051 & 0,052 & 0,035 & 0,018 \\
& & & & $(0,043)$ \\
Doutorado & 0,074 & 0,078 & 0,034 & 0,044 \\
& & & & $(0,051)$ \\
Tempo de Empresa (em anos) & 15,83 & 16,22 & 11,55 & 4,52 \\
Tempo no Cargo (em anos) & $(12,64)$ & $(12,79)$ & $(10,01)$ & $(2,42)$ \\
Percentual de Mulheres & 10,00 & 10,25 & 7,21 & 2,84 \\
\# de observações & $(9,73)$ & $(9,87)$ & $(7,66)$ & $(1,87)$ \\
\hline
\end{tabular}

Nota. ${ }^{1}$ Entre parênteses, o desvio padrão quando necessário.

Os modelos Probit são adequados para a análise de dados em que a variável resposta é qualitativa e binária. Nosso objetivo é aferir a probabilidade de uma mulher ocupar ou não o cargo mais elevado na hierarquia da empresa. Entretanto os dados permitem somente a verificação do que já ocorreu. Em nosso caso, observamos o fato do cargo de CEO nas empresas ser ocupado ou não por uma mulher, e não a chance (ou probabilidade) que todas as mulheres tiveram para chegar a esse cargo. Sendo assim, construímos uma variável denominada CEO Mulher, e esta variável assume o valor 1 quando uma mulher é a CEO das empresas, e zero para os casos em que o CEO é um homem.

O modelo Probit pode ser definido pela seguinte equação:

$$
\operatorname{Pr}(Y=1 \mid x)=\Phi\left(x_{i} \beta\right)
$$


Onde $\Phi$ é a função de distribuição cumulativa de uma normal e $\beta$ representa o vetor dos parâmetros estimados neste modelo.

Neste estudo, estimaremos a seguinte equação:

$$
\operatorname{Pr}(\text { CEO Mulher })=\alpha+\sum \beta_{\mathrm{i}} \text { Setor }_{\mathrm{i}}+\sum \delta_{\mathrm{j}} \text { Região }_{\mathrm{j}}+\sum \gamma_{\mathrm{k}} \mathrm{X}_{\mathrm{k}}+\varepsilon
$$

Onde:

Setor é a representação dos setores econômicos (agronegócios, indústria, comércio e serviços), sendo agronegócios a dummy excluída.

Região é a representação das regiões geográficas (Norte, Nordeste, Sul, Sudeste e CentroOeste), sendo excluída a região Sudeste.

$\boldsymbol{X}_{k}$ representam as características das empresas em quatro variáveis: receita líquida (em logaritmo), uma variável dummy para empresas que têm um Conselho de Administração ativo, idade da empresa (em anos) e uma variável com o percentual do controle acionário da empresa que está em poder de seus executivos.

Na próxima seção, discutiremos essa abordagem inicial e as possibilidades de análise a partir dela.

\section{Resultados Empíricos}

A partir do modelo especificado pela equação (1), utilizamos os dados das empresas para estimar a equação. Dos aspectos inicialmente analisados, podemos perceber na Tabela 3 que somente o indicador para existência de um Conselho Administrativo da empresa teria impacto significativo na escolha do gênero do CEO.

Tabela 3

Modelo básico, segundo Equação (1)

\begin{tabular}{lcc}
\hline & Coeficiente Estimado & Efeito Marginal \\
\hline Indústria & $-0,359$ & \\
Serviços & $-0,114$ & \\
Sul & 0,105 & \\
Nordeste & 0,395 & \\
Centro-Oestre & 0,036 & $-12,15 \%$ \\
Idade da Empresa & 0,007 & \\
Ln(Receita) & $-0,026$ & \\
Tem Conselho Administrativo & $-0,674^{*}$ & \\
Percentual de Controle Acionário por Executivos & 0,002 & \\
Constante & $-0,911$ & \\
Número de Observações & 222 & \\
Pseudo R & 0,0731 & \\
\hline
\end{tabular}

Nota. O símbolo de * indica que a variável é significante à $1 \%$. Todas as demais variáveis não apresentaram impacto significante estatisticamente. 
Analisando o efeito marginal dessa variável na média, podemos afirmar que a existência de um Conselho Administrativo diminui em 12,15\% as chances de o CEO da empresa ser uma mulher.

Apesar das outras variáveis incluídas no modelo terem potencial para explicar a escolha do CEO, elas não se mostraram significativas nessa estimação. Assim, procuramos uma alternativa de estimação, utilizando perfis de empresas mais similares. O caminho escolhido foi a divisão das empresas em dois tipos distintos: empresas nas quais o controle acionário está totalmente em poder de seus executivos e outras nas quais os executivos não têm o poder acionário completo. Essa divisão possibilita que a análise seja diferenciada para empresas com o corpo executivo menos profissionalizado - aquelas nas quais os executivos têm total controle acionário - e as mais profissionais. A divisão é embasada no estudo de Barth, Gulbrandsen e Schone (2005), no qual os autores traçam a relação entre controle acionário e profissionalização de gestores com a produtividade na empresa.

Embora não seja possível inferir pelos dados da pesquisa se as empresas são familiares ou não, acreditamos que o alto percentual acionário nas mãos dos gestores é uma proxy adequada para tal. Os resultados para essas estimativas estão na Tabela 4.

Os resultados mostram que, para os casos em que o controle acionário é totalmente retido por seus executivos, três variáveis apresentam relevância: setor de atuação da empresa, posição geográfica e existência de um Conselho Administrativo. O setor industrial diminui em $12 \%$ as chances de uma mulher ocupar o cargo de CEO de uma empresa, enquanto a instalação da empresa na região Sul do país aumenta em 15,53\% as chances dessa ocorrência. A presença de um Conselho Administrativo, assim como nos resultados da Tabela 3, também diminui em 12,76\% a probabilidade de uma mulher tornar-se CEO de acordo com Controle Acionário.

Tabela 4

\section{Modelos alternativos}

\begin{tabular}{lcccc}
\hline & \multicolumn{2}{c}{$\begin{array}{c}\text { 100\% do Controle Acionário } \\
\text { com Executivos }\end{array}$} & \multicolumn{2}{c}{$\begin{array}{c}\text { Controle Acionário não é } \\
\text { totalmente de seus Executivos }\end{array}$} \\
& $\begin{array}{c}\text { Coeficiente } \\
\text { Estimado }\end{array}$ & $\begin{array}{c}\text { Efeito } \\
\text { Marginal }\end{array}$ & $\begin{array}{c}\text { Coeficiente } \\
\text { Estimado }\end{array}$ & $\begin{array}{c}\text { Efeito } \\
\text { Marginal }\end{array}$ \\
\hline Indústria & $-1,260^{*}$ & $-12,00 \%$ & $-0,040$ & \\
Serviços & $-0,551$ & & $-0,120$ & \\
Sul & $0,840^{*}$ & $15,53 \%$ & $-0,449$ & \\
Nordeste & $-0,051$ & & 0,324 & \\
Centro-Oestre & 0,374 & & $-0,177$ & $-1,40 \%$ \\
Idade da Empresa & 0,002 & & 0,002 & $-8,89 \%$ \\
Ln(Receita) & 0,125 & & $-0,121^{*}$ & \\
Tem Conselho Administrativo & $-0,799^{*}$ & $-12,76 \%$ & $-0,643$ & \\
Constante & $-1,684$ & & 0,199 & \\
Número de Observações & 99 & & 193 & \\
Pseudo R & 0,1555 & & 0,1319 & \\
\hline
\end{tabular}

Nota. O símbolo de * indica que a variável é significante a $10 \%$, ** a $5 \%$ e. Todas as demais variáveis não apresentaram impacto estatístico significante.

Quando o controle acionário não pertence totalmente a seus executivos, a administração da empresa tende a ser mais profissional, mas o impacto da existência de um Conselho de Administração permanece negativo, agora diminuindo em $9 \%$ as chances de uma mulher assumir o cargo de CEO. O 
tamanho da empresa, porém, também passa a ser relevante. Empresas com maior receita tendem a diminuir em $1,4 \%$ a probabilidade de terem como CEO uma mulher.

Este segundo fator pode estar relacionado à inércia da cultura com relação ao risco em geral e também com relação aos gêneros (Audia \& Greve, 2006, mostram que empresas maiores são mais inertes com relação ao risco do que as pequenas), mas também pode refletir a menor presença de mulheres em empresas de maior porte que possam ser conduzidas a esse cargo.

O resultado interessante em todas as regressões é a regularidade com que a presença de um Conselho de Administração em determinada empresa tende a diminuir a probabilidade de uma mulher tornar-se sua CEO. Em consonância com Westphal e Fredrickson (2001), nossos resultados também apontam a importância de o Conselho reconhecer no potencial CEO características próprias dos conselheiros e, assim, terem confiança suficiente para indicá-lo ao cargo. Na base de dados utilizada, não temos possibilidade de analisar a composição do conselho; mas a baixa presença de mulheres em cargos de liderança no Brasil sugere que os conselhos sejam formados majoritariamente por homens, o que corrobora o que foi encontrado pelo autores. Assim, a baixa identificação de um Conselho com uma candidata a CEO devido às diferenças naturais de gênero pode influenciar negativamente na sua escolha para o cargo.

\section{Conclusões e Pesquisas Futuras}

O presente estudo analisa a possibilidade de existência de teto de vidro para a promoção de mulheres ao cargo de CEO em empresas no Brasil. Utilizando uma base de dados com informações financeiras e de gestão das empresas, podemos concluir que as mulheres têm ascensão mais restrita ao cargo de CEO, caso a empresa em que trabalhem tenha um Conselho de Administração constituído.

A existência de um Conselho de Administração teoricamente implicaria maior distanciamento entre os interesses pessoais e os interesses da empresa. O Conselho tomaria decisões baseadas na eficiência e na melhoria da perspectiva de lucratividade da empresa. Entretanto, estudos anteriores ao nosso (como Lee \& James, 2007; Westphal \& Fredrickson, 2001) mostram que as implicações de escolha dos representantes das empresas, os CEOs, levam em conta características dos decisores. Ou seja, para um grupo composto majoritariamente por homens, a escolha de CEO mulher pode ser menos provável - e nosso estudo diz que é em torno de $12 \%$ menos provável - pois esta não necessariamente passa a imagem aos membros do Conselho de seu alinhamento com suas propostas e características. Ao mesmo tempo, a preocupação do Conselho em ter uma boa imagem para o público em geral pode afetar a escolha do gênero do CEO. Lee e James (2007) mostram que os reflexos na precificação das ações para empresas que anunciam CEO do gênero feminino são mais negativos do que para as que anunciam CEO homens.

Desse modo, a escolha de CEO mulher, muitas vezes, está ligada ao fato de que a empresa e seu Conselho pretendem mostrar algum fator adicional para os investidores em geral, que não somente a adequação do indivíduo ao posto que ocupa. Uma clara limitação de nosso estudo é a composição da base de dados, que impede a análise da composição do Conselho das empresas abordadas; entretanto, sabendo que a composição dos Conselhos de Administração é majoritariamente masculina na totalidade das empresas existentes, admitimos que a mesma métrica é utilizada nas empresas estudadas.

Nosso estudo corrobora as conclusões encontradas em relação a outros países e motiva a continuação da abordagem de gênero ligada aos resultados financeiros da empresa. Uma direção para estudo futuro é um aprofundamento das características do Conselho de Administração - perfis e regras para escolha de CEOs - em empresas. Uma segunda possibilidade seria a análise da remuneração e da sua composição para CEOs no Brasil. Ambas as possibilidades são promissoras e não puderam ser abordadas no momento, devido à restrição de informações na base de dados disponível; mas 
acreditamos que os atuais resultados possam motivar a confecção de bases de dados confiáveis que permitam análises como essas.

Artigo recebido em 21.07.2009. Aprovado em 16.07.2010.

\section{Agradecimentos}

A autora agradece o excelente trabalho da assistente de pesquisa Carolina Flores Gomes e o apoio financeiro do CNPq por meio da bolsa de produtividade de pesquisa no. 307513/2007-6.

\section{Notas}

${ }^{1}$ Para fins de comparação, a participação dos homens no mercado de trabalho é estável no período e em torno dos $70 \%$.

${ }^{2}$ Não necessariamente o cargo mais elevado da empresa tem a denominação de CEO, mas utilizaremos esta sigla para fins de maior simplicidade nas explicações.

${ }^{3} \mathrm{O}$ desvio padrão desta variável é bastante elevado. Um dos motivos para isso é uma determinada empresa com receita líquida anula de $\mathrm{R} \$ 19.159 .545$ mil por ano (enquanto a empresa com a menor receita anual líquida tem valor $\mathrm{R} \$ 16$ mil por ano).

${ }^{4}$ Estes dados foram capturados por meio da pergunta "Qual o cargo mais elevado na hierarquia da empresa?", que permitia uma resposta aberta do entrevistado. 61\% das empresas declararam ser Presidente, Diretor ou Diretor-Presidente. Foram excluídos nomes de cargos que refletiam não ser o de mais elevada hierarquia (por exemplo, assessor de diretoria).

${ }^{5}$ Uma possibilidade seria a análise das características dos CEOs, que fizeram com que fossem escolhidos para tal posto. Entretanto, para a análise ser adequada, seriam necessários dados de outros indivíduos que trabalharam na empresa e não foram selecionados para o cargo. Adicionalmente, inserir as características dos CEOs na regressão com as informações das empresas também foi testado; mas nenhuma variável foi significante para alteração da probabilidade do gênero do CEO. A explicação recai na grande semelhança entre as amostras de CEOs homens e mulheres. Os resultados estão disponíveis mediante solicitação ao autor.

\section{Referências}

Audia, P., \& Greve, H. (2006). Less likely to fail: low performance, firm size, and factory expansion in the shipbuilding industry. Management Science, 52(1), 83-94. doi: 10.1287/mnsc.1050.0446

Barth, E., Gulbrandsen, T., \& Schone, P. (2005). Family ownership and productivity: the role of owner-management. Journal of Corporate Finance, 11(1-2), 107-127.

Becker, G. (1965). A theory of the allocation of time. Economic Journal, 75(299), 493-517.

Berger, L. M., \& Waldfogel, J. (2004). Maternity leave and the employment of new mothers in the United States. Journal of Population Studies, 17(2), 331-349.

Bjerk, D. (2008). Glass ceilings or sticky floors? Statistical discrimination in a dynamic model of hiring and promotion. Economic Journal, 118(530), 961-982.

Blau, F., \& Devaro, J. (2007). New evidence on gender differences in promotion rates: an empirical analysis of a sample of new hires. Industrial Relations, 46(3), 511-550.

Bourdieu, P. (2009). A dominação Masculina (M. H. Kuhener Trad.). Rio de Janeiro: Bertrand Brasil. (Obra original publicada em 1990). 
Carvalho, S. S., Firpo, S., \& Gonzaga, G. (2006). Os efeitos da licença maternidade sobre salários e emprego da mulher no Brasil. Política e Planejamento Econômico, 36(3), 489-523.

Giuberti, A. C., \& Menezes, N. A., Filho (2005). Discriminação de rendimentos por gênero: uma comparação entre o Brasil e os Estados Unidos. Economia Aplicada, 9(3), 369-384.

Gordo, L. R. (2009). Why are women delaying motherhood in Germany? Feminist Economics, 15(4), 57-75.

Guimarães, L. (2008, maio 11). O teto de vidro blindado. Jornal o Estado de São Paulo. Recuperado em 8 abril, 2009, de http://www.estadao.com.br/suplementos/not_sup170706,0.htm

Hultin, M. (2003). Some take the glass escalator, some hit the glass ceiling? Work \& Occupations, $30(1), 30-61$.

Jones, D., \& Makepeace, G. (1996). Equal worth, equal opportunities: pay and promotion in an internal Labour market. The Economic Journal, 106(435), 401-409.

Kaufman, B. E., \& Hotchkiss, J. L. (2003) The economics of labor markets (6th ed.). Ohio: Thomson Learning.

Lee, P. M., \& James, E. H. (2007). She'-e-os: gender effects and investor reactions to the announcements of top executive appointments. Strategic Management Journal, 28(3), 227-241.

Madalozzo, R. (2009). Occupational segregation and the gender wage gap in Brazil: an empirical analysis. Economia Aplicada, 14(2), 147-168.

Morrison, A. M., White, R. P., Velsor, E. van, \& The Center for Creative Leadership. (1987). Breaking the glass ceiling: can women reach the top of America's largest corporations? Reading, MA: Addison-Wesley.

Napari, S. (2010). Is there a motherhood wage penalty in the finnish private sector? Review of Labour Economics \& Industrial Relations, 24(1), 55-73.

Powell, G. N., \& Butterfield, D. A. (1994). Investigation of the "glass ceiling" phenomenon: an empirical study of actual promotions to top management. Academy of Management Journal, $37(1), 68-86$.

Santos, R. V., \& Ribeiro, E. P. (2006). Diferenciais de rendimentos entre homens e mulheres no Brasil revisitado: explorando o "teto de vidro". Recuperado em 23 abril, 2009, de http://www.ie.ufrj.br/eventos/seminarios/pesquisa/texto06_05_02.pdf

Westphal, J. D., \& Fredrickson, J. W. (2001). Who directs strategic change? Director experience, the selection of new CEOs, and change in corporate strategy. Strategic Management Journal, 22(12), 1113-1137.

Wolfers, J. (2006). Diagnosing discrimination: stock returns and CEO gender [Working Paper $\mathrm{N}^{\circ}$ 11989]. National Bureau of Economic Research, Cambridge, MA. 